\title{
Thyroid functions in patients with bipolar disorder and the impact of quetiapine monotherapy: a retrospective, naturalistic study
}

This article was published in the following Dove Press journal:

Neuropsychiatric Disease and Treatment

\author{
Chao $\mathrm{Li}^{1-3, *}$ \\ Jianbo Lai ${ }^{1,4,5, *}$ \\ Tingting Huang ${ }^{2}$ \\ Yuqing $\mathrm{Han}^{2}$ \\ Yanli Du ${ }^{2}$ \\ Yi Xu ${ }^{1,4,5}$ \\ Shaohua $\mathrm{Hu}^{1,4,5}$ \\ 'Department of Psychiatry, First Affiliated \\ Hospital, Zhejiang University School of \\ Medicine, Hangzhou 310003, People's \\ Republic of China; ${ }^{2}$ Department of \\ Clinical Medicine, Zhejiang University \\ School of Medicine, Hangzhou 310003, \\ People's Republic of China; ${ }^{3}$ Department \\ of Psychiatry, Ningbo Psychiatric Hospital \\ of Zhejiang Province, Ningbo 315000, \\ People's Republic of China; ${ }^{4}$ The Key \\ Laboratory of Mental Disorder's \\ Management in Zhejiang Province, \\ Hangzhou 310003, People's Republic of \\ China; ${ }^{5}$ Brain Research Institute of \\ Zhejiang University, Hangzhou 310003 , \\ People's Republic of China \\ *These authors contributed equally to \\ this work
}

\begin{abstract}
Objective: Previous studies have demonstrated a potentially close relationship between mood disorders and thyroid abnormalities. The aims of this study are to investigate: 1) whether significant differences of thyroid profiles exist between manic and depressive episodes in patients with bipolar disorder (BD); 2) the impact of quetiapine monotherapy on thyroid functions in depressed BD patients.

Methods: In this retrospective naturalistic study, we reviewed patients' information based on an electronic medical system from January 2015 to April 2019. Patients with a discharge diagnosis of BD, a current depressive or manic episode, were screened. All depressed BD patients were treated with quetiapine monotherapy for at least one month. For all patients enrolled, the demographic, clinical data and thyroid functions were recorded. The differences between thyroid profiles including total thyroxine (TT4), total triiodothyronine (TT3), free triiodothyronine (FT3), free thyroxine (FT4) and thyroid-stimulating hormone (TSH) between patients with different episodes were analyzed. In addition, the change of thyroid functions before and after one-month or three-month quetiapine treatment in depressed BD patients was also analyzed.
\end{abstract}

Results: Totally, 28 patients with a manic episode and 58 patients with a depressive episode were enrolled. No significant differences in thyroid profiles were found in patients with different mood episodes. After one-month quetiapine treatment, serum levels of TT4, FT4 and FT3 were significantly reduced $(P<0.05)$, TSH was increased $(P=0.015)$, while TT3 was not significantly changed $(P=0.425)$. After three-month quetiapine treatment, serum levels of TT4, TT3, FT4 and FT3 were significantly reduced $(P<0.05)$, except TSH $(P=0.990)$.

Conclusion: These findings indicate that thyroid functions were not significantly fluctuated between depressive and manic episodes in BD patients. Nonetheless, quetiapine treatment may disturb the thyroid system and needs close monitoring.

Keywords: thyroid function, bipolar disorder, quetiapine, mania

\section{Introduction}

Bipolar disorder (BD) is a common psychiatric disorder, characterized by recurrent fluctuations in mood states and energy, with significantly increased rates of morbidity and mortality. ${ }^{1,2}$ The etiology of this disease is multi-factorial and complicated. Previous researches have shown that patients with BD are 2.55 times more likely to be associated with thyroid dysfunction. ${ }^{3}$ Thyroid autoimmunity even appears to be an independent risk factor of BD. ${ }^{4}$ Moreover, disturbances of thyroid functions, whether inadequate (hypothyroidism) or excessive (hyperthyroidism), can possibly lead to
Department of Psychiatry, The First Affiliated Hospital, Zhejiang University School of Medicine, 79 Qingchun Road, Hangzhou 310003, People's Republic of China

Tel +865 718 7235948

Fax +86 57187235948

Email dorhushaohua@zju.edu.cn 
mood swings and anxiety. ${ }^{5-7}$ However, the differences of thyroid profiles exist between manic (or hypomanic) and depressive episodes were still not conclusive.

The most common causes of thyroid dysfunction are congenital, autoimmune and iatrogenic. ${ }^{8,9}$ In clinical practice, drug-induced thyroid dysfunctions are not rare, targeting at various pathways of synthesis, secretion, transport, metabolism and absorption of thyroid hormones. ${ }^{10}$ A previous naturalistic study indicated that the use of antipsychotics was associated with lower concentration of serum-free thyroxine (FT4) in medicated patients, when compared to healthy individuals. ${ }^{11}$

Quetiapine, one of the second-generation antipsychotics, is commonly used to manage acute depressive phase of $\mathrm{BD} .{ }^{12} \mathrm{~A}$ multicenter, double-blind, randomized, placebo-controlled study has shown significant elevation of thyroid-stimulating hormone (TSH) in depressed pediatrics and adolescences with $\mathrm{BD}$, after receiving 8-week treatment of quetiapine extendedrelease (dose range, $150-300 \mathrm{mg} /$ day) ${ }^{13} \mathrm{In}$ another prospective study, 6-week treatment of quetiapine (average dose, 407 $\mathrm{mg}$ /day) in schizophrenic patients led to a modest decrease in total thyroxine (TT4) and a small decrease in total triiodothyronine (TT3). ${ }^{14}$ However, these changes in thyroid functions were thought to be not clinically significant, because of no alterations in TSH and absence of relevant symptoms. ${ }^{14}$ To date, no published study has ever reported the acute change of thyroid profiles following quetiapine.

To address these issues, the aim of the current study is to investigate (1) whether significant differences of thyroid profiles exist between manic and depressive episodes in BD patients; and (2) the impact of quetiapine monotherapy on thyroid functions in depressed BD patients.

\section{Methods}

This study was approved by the Institute Review Board of the First Affiliated Hospital, Zhejiang University School of Medicine. Due to the retrospective, non-interventional and anonymous nature of this study, informed consent from patients was waived.

Based on the hospital electronic medical system, naturalistic medical information of in-patients in the First Affiliated Hospital, Zhejiang University School of Medicine, was extracted from January 2015 to April 2019. Inclusion criteria were: 1) 15 55 years old; 2) drug-naïve or medication-free for at least 3 months before hospitalization; 3) a discharge diagnosis with BD, a depressive episode or a manic episode, which was made according to the Diagnostic and Statistical Manual of Mental Disorder fifth edition
(DSM-5); and 4) for patients with a depressive episode, receiving quetiapine monotherapy during hospitalization and were followed up for at least one month. For depressed patients, the maintenance dose of quetiapine was restricted to 300 500 mg per day. The exclusion criteria include: 1) comorbid with other psychiatric disorders; 2) severe physical illnesses that need medications; 3) any known autoimmune diseases; and 4) intolerable to quetiapine therapy.

For all patients enrolled, demographic data (eg, gender, age, height, weight and disease course) and laboratory tests of thyroid functions (eg, TT4, FT4, TT3, FT3 and TSH) were collected. Body mass index (BMI) was defined as the weight divided by the square of the height $\left(\mathrm{kg} / \mathrm{m}^{2}\right)$. For patients with a depressive episode, thyroid functions and results of clinical assessments (24-item Hamilton Depression Rating Scale and Hamilton Anxiety Rating Scale) before and after one-month quetiapine treatment were recorded. For patients with a manic episode, only baseline thyroid functions were recorded.

All statistical analyses were performed with the Statistical Package for the Social Sciences software v.19.0 (SPSS Inc, Chicago, IL). Patients at baseline were divided into two groups: the mania group and depressive group. For patients in the depressive group, we compared the thyroid functions between baseline and post-treatment. The categorical data were analyzed by Pearson Chi-square test. Continuous variable data were analyzed by pairedsample $t$-test (normally distributed data) or Mann-Whitney U-test (not normally distributed data). Normally distributed data are presented as mean \pm standard deviation (SD); $P$-value $<0.05$ was denoted as statistical significance.

\section{Results}

\section{Demographic and clinical data}

Totally, 86 patients diagnosed with $\mathrm{BD}$ were eligible in this study (28 patients in the manic group and 58 in the depressive group). Manic patients were significantly older than depressed patients $(P<0.001)$. The duration of the current episode was longer in depressed patients $(P<0.05)$. No difference was found in gender, weight, height, BMI and the total disease course between the two groups (see Table 1).

\section{Baseline thyroid functions in manic and depressed BD patients}

In the manic group, the thyroid profiles of all patients were within the normal limits. However, in the depressed group, 
Table I Demographic and clinical characteristics of the study subjects (mean \pm SD)

\begin{tabular}{|l|l|l|l|}
\hline & Manic group $\mathbf{n = 2 8}$ & Depressive group $\mathbf{n = 5 8}$ & P-value* \\
\hline Gender (n, male/female) & $15 / 13$ & $31 / 27$ & 0.992 \\
Age (years) & $29.5 \pm 8.9$ & $20.7 \pm 6.7$ & $<0.00 \mathrm{I}$ \\
Height (centimeter) & $167.6 \pm 10.2$ & $167.6 \pm 8.1$ & 0.984 \\
Weight (kilogram) & $63.13 \pm 14.1$ & $60.93 \pm 15.2$ & 0.530 \\
BMI (kg/m ${ }^{2}$ ) & $22.26 \pm 3.37$ & $21.51 \pm 4.20$ & 0.419 \\
Total disease course (month) & $31.02 \pm 28.87$ & $48.02 \pm 61.77$ & 0.128 \\
Duration of current episode (day) & $23.8 I \pm 22.91$ & $137.61 \pm 211.98$ & 0.004 \\
\hline
\end{tabular}

Note: *Significant at $P<0.05$ (two-tailed).

Abbreviation: SD, standard deviation.

the baseline TSH value of three patients was lower than the normal below limit, and for one patient it was higher than the normal upper limit. As shown in Table 2, no difference of baseline thyroid profiles, including TT4, FT4, TT3, FT3 or TSH, was identified between the two groups.

\section{Impact of quetiapine treatment on thyroid functions in depressed BD patients}

In the depressed group, the maintenance dose of quetiapine in three patients was $500 \mathrm{mg}$ per day, another three was $400 \mathrm{mg}$ per day and the remaining $(\mathrm{n}=52)$ was all 300 mg per day. After one-month quetiapine monotherapy, the rating scores of HAMD-24 and HAMA were significantly decreased $(P<0.05)$ (see Table 3$)$. The TSH value of two patients was over the normal upper limit and reached the diagnosis of subclinical hypothyroidism. Notably, serum values of TT4, FT4 and FT3 were significantly reduced after treatment $(P<0.05)$, TSH was increased $(P=0.015)$, while TT3 was not significantly changed $(P=0.425)$ (see Figure 1).

In addition, 13 depressed patients receiving quetiapine were followed up for three months. Serum TSH in two patients was over the normal upper limit and reached the diagnosis of subclinical hypothyroidism. In these patients, only serum level of TT4 was reduced after one-month treatment $(P=0.007)$. However, following three-month treatment, serum level of TT4, TT3, FT4 and FT3 was significantly reduced compared to baseline $(P<0.05)$, and serum level of TSH was not significantly changed $(P=0.990)$ (see Figure 2).

\section{Discussion}

In the present study, we retrospectively reviewed the differences of thyroid functions between manic and depressive episodes in BD patients and explored the change of thyroid profiles following quetiapine treatment in the depressed patients. The findings indicated that thyroid profiles were not significantly different between different mood episodes, but could be remarkably influenced by short-term quetiapine treatment. Although the alteration in thyroid function was not clinically significant in most patients, the findings in the current study still warrant more attention.

Early studies have reported decreased TT3 and TT4, but not TSH, in patients receiving 6-week quetiapine treatment. ${ }^{14} \mathrm{~A}$ series of case reports also presented quetiapine-associated hypothyroidism. ${ }^{15-18}$ In a recent naturalistic study, lower FT4 was also associated with the use of

Table 2 Compassion of baseline thyroid profiles in patients with manic and depressive episodes (mean \pm SD)

\begin{tabular}{|l|l|l|l|l|}
\hline & Manic group $\mathbf{n = 2 8}$ & Depressive group $\mathbf{n = 5 8}$ & Reference range & P-value* \\
\hline TT4 $(\mathrm{nmol} / \mathrm{L})$ & $97.18 \pm 24.09$ & $96.02 \pm 18.30$ & $55.47 \sim 161.25$ & 0.823 \\
TT3 $(\mathrm{nmol} / \mathrm{L})$ & $1.48 \pm 0.36$ & $1.52 \pm 0.30$ & $1.02 \sim 2.96$ & 0.619 \\
TSH $(\mathrm{mU} / \mathrm{L})$ & $1.50 \pm 0.62$ & $1.53 \pm 1.02$ & $0.38 \sim 4.34$ & 0.893 \\
FT4 $(\mathrm{pmol} / \mathrm{L})$ & $15.55 \pm 3.50$ & $16.27 \pm 2.59$ & $10.45 \sim 24.38$ & 0.287 \\
FT3 $(\mathrm{pmol} / \mathrm{L})$ & $4.72 \pm 0.70$ & $4.96 \pm 0.74$ & $2.77 \sim 6.31$ & 0.154 \\
\hline
\end{tabular}

Note: *Significant at $P<0.05$ (two-tailed).

Abbreviation: SD, standard deviation; TT4, total thyroxine; TT3, total triiodothyronine; FT3, free triiodothyronine; FT4, free thyroxine; TSH, thyroid-stimulating hormone. 
Table 3 Changes of scale scores before and after one-month quetiapine treatment in depressed patients with bipolar disorder (mean $\pm \mathrm{SD})$

\begin{tabular}{|l|l|l|l|}
\hline & Baseline $\mathbf{n = 5 8}$ & Post-treatment $\mathbf{n = 5 8}$ & P-value* \\
\hline HAMD-24 & $29.63 \pm 9.89$ & $13.88 \pm 10.12$ & $<0.001$ \\
HAMA & $22.09 \pm 8.17$ & $11.18 \pm 8.24$ & $<0.001$ \\
\hline
\end{tabular}

Note: *Significant at $P<0.05$ (two-tailed).

Abbreviations: SD, standard deviation; HAMD-24, 24-item Hamilton Depression Rating Scale; HAMA, Hamilton Anxiety Rating Scale.
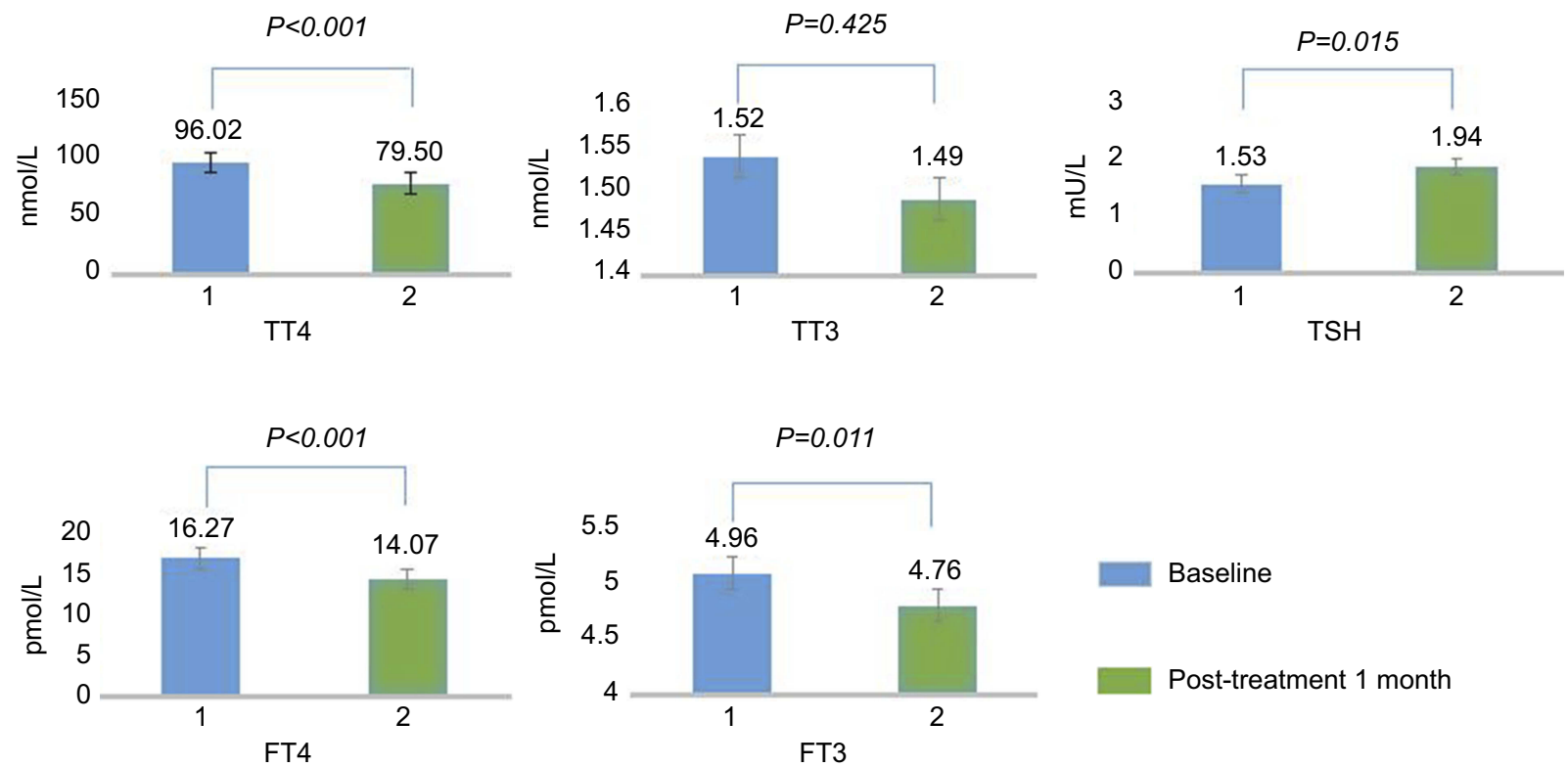

Baseline

Post-treatment 1 month

Figure I Changes of thyroid indices before and after one-month quetiapine treatment in depressed patients with bipolar disorder ( $\mathrm{n}=58$ ).

Abbreviations: TT4, total thyroxine; TT3, total triiodothyronine; FT3, free triiodothyronine; FT4, free thyroxine; TSH, thyroid-stimulating hormone.
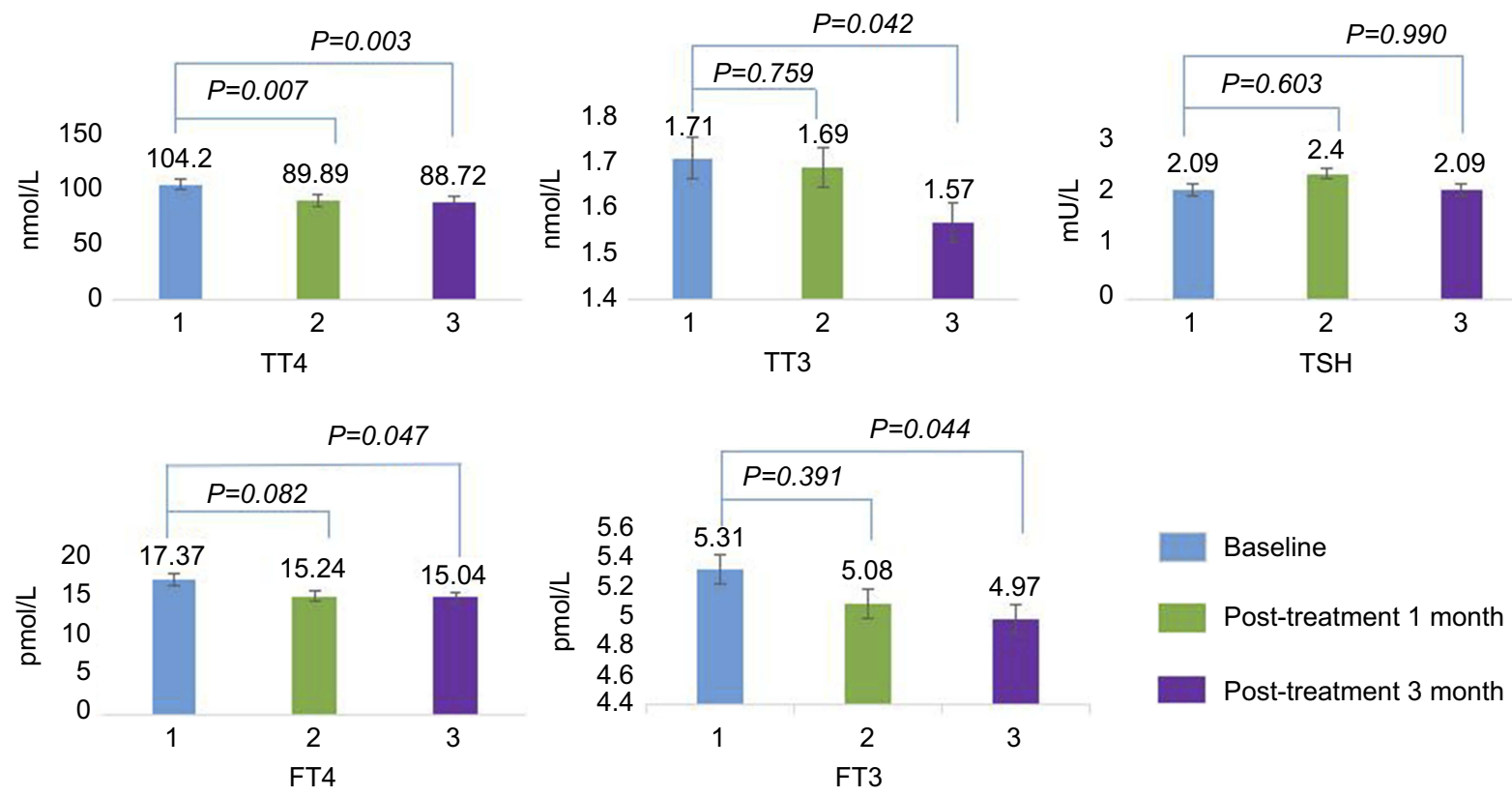

Baseline

Post-treatment 1 month

Post-treatment 3 month

Figure 2 Changes of thyroid indices before and after one- or three-mouth quetiapine treatment in depressed patients with bipolar disorder ( $\mathrm{n}=13$ ). Abbreviations: TT4, total thyroxine; TT3, total triiodothyronine; FT3, free triiodothyronine; FT4, free thyroxine; TSH, thyroid-stimulating hormone. 
antipsychotics (including quetiapine). ${ }^{11}$ However, these changes in thyroid functions rarely resulted in clinical symptoms and were not fully concerned in clinical practice. Due to the lack of research findings, it is not clear whether these changes of thyroid profiles are reversible or persistent. Nonetheless, this subtle alteration in thyroid indices could be clinically significant. As shown in previous studies, adjuvant levothyroxine therapy might accelerate treatment response in patients with bipolar depression. ${ }^{19}$ Therefore, the reduction of TT4, FT4 and FT3 caused by quetiapine may delay the clinical treatment response. ${ }^{19}$ Given the close relationship between thyroid hormones and body metabolism, the subtle reduction in TT4, FT4 and FT3 may also associate with weight gain following quetiapine treatment. In our study, the findings also revealed statistically significant changes in thyroid functions and support that quetiapine may disrupt the thyroid functions. However, few patients met the diagnostic criteria for hypothyroidism or subclinical hypothyroidism after treatment. In addition, the duration of these changes in thyroid profiles may be not long enough to cause any clinical changes of BD.

The mechanisms underlying quetiapine-associated thyroid abnormalities remain to be clarified. Some evidences suggested a direct or indirect action of quetiapine on the hypothalamus-pituitary-thyroid axis. Quetiapine is an atypical antipsychotic drug with dopamine $\mathrm{D}_{2}$ receptor and serotonin $5-\mathrm{HT}_{2 \mathrm{~A}}$ receptor antagonizing activity. ${ }^{20}$ Dopamine can inhibit the pituitary secretion of thyrotropin-releasing hormone (TRH). ${ }^{21}$ Therefore, blockage of dopamine receptor by quetiapine may enhance dopamine's effect on the pituitary and decrease its releasing of TRH, eventually resulting in the reduction of T3 and T4. In addition, the metabolism of thyroid hormones by UDP-glucuronosyl transferase could be interfered due to quetiapine treatment. ${ }^{22}$ UDP-glucuronosyl transferase has participated in the glucuronidation of thyroid hormones, and certain antipsychotics as well. ${ }^{23}$ Quetiapine might compete for the metabolism of thyroid hormones in Phase II glucuronidation, which was possibly responsible for the changes of thyroid hormones. ${ }^{22}$ In addition, some studies reported an altered level of anti-thyroid peroxidase autoantibodies following quetiapine, which indicated a potential autoimmune pathway involved in the quetiapine-associated changes in thyroid indices. ${ }^{18}$

Furthermore, no significant differences in thyroid parameters were found between manic and depressive episodes. To the best of our knowledge, our study is the first to compare thyroid functions in BD patients with different episodes. This finding indicated that the thyroid functions may not be a determinant factor for mood status, and mood swings have no apparent influence on the thyroid functions. Previous studies have reported a close relationship between thyroid disturbance and the development of BD. ${ }^{3,4}$ Interestingly, the association between mood dysregulation and thyroid functions may be more apparent in adults, but not in youths. ${ }^{24}$ Together with our findings, these researches indicate the influence of quetiapine on thyroid hormones is likely to have individual differences. It may be not the absolute values of thyroid indices, but their changes in serum concentrations, that causes mood swings. In future studies, thyroid data from euthymic BD patients and healthy subjects are warranted to better clarify the associations between BD and thyroid functions.

This study has several limitations to be noted. First, the retrospective design and the relative small sample size limited the stringency of the current study. However, the diagnosis of BD was made according to DSM-5 during hospitalization. All depressed patients enrolled received naturalistic medical interventions. The alterations in thyroid functions reported herein are still credible and warrant more clinical attention. Second, the associations between parameters of thyroid functions and the illness duration, episode number and severity of symptom were not investigated and analyzed in this study, and these factors may also influence the results of thyroid tests. Third, although the study compared the change of thyroid hormones before and after quetiapine treatment, our study still failed to confirm whether these changes were temporary or persistent. Meanwhile, the clinical indications of these changes and their long-term influence on the patients were also not clear. At last, the weight of these patients after quetiapine treatment was not recorded, and we cannot analyze the association between quetiapine-induced thyroid dysfunction and change in weight.

\section{Conclusion}

This study further provides evidences that quetiapine treatment in BD patients may disturb thyroid metabolism even at the early stage of pharmacotherapy. Integrated psychiatric and endocrine care is needed in this population.

\section{Acknowledgment}

This work was partly supported by the grants of the National Key Basic Research Program (2016YFC1307100, 2016YFC1307104), National Clinical Research Center for Mental Health Disorders (2015BAI13B02) and the Key Research Project of Zhejiang Province (2015C03040). 


\section{Disclosure}

The authors report no conflicts of interest in this work.

\section{References}

1. Grande I, Berk M, Birmaher B, Vieta E. Bipolar disorder. Lancet. 2016;387(10027):1561-1572. doi:10.1016/S0140-6736(15)00241-X

2. Alonso J, Petukhova M, Vilagut G, et al. Days out of role due to common physical and mental conditions: results from the WHO world mental health surveys. Mol Psychiatry. 2011;16(12):12341246. doi: $10.1038 / \mathrm{mp} .2010 .101$

3. Krishna VN, Thunga R, Unnikrishnan B, et al. Association between bipolar affective disorder and thyroid dysfunction. Asian J Psychiatr. 2013;6(1):42-45. doi:10.1016/j.ajp.2012.08.003

4. Barbuti M, Carvalho AF, Kohler CA, et al. Thyroid autoimmunity in bipolar disorder: a systematic review. J Affect Disord. 2017;221:97106. doi:10.1016/j.jad.2017.06.019

5. Vogel A, Elberling TV, Hørding M, et al. Affective symptoms and cognitive functions in the acute phase of graves' thyrotoxicosis. Psychoneuroendocrinology. 2007;32(1):36-43. doi:10.1016/j.psyneu en.2006.09.012

6. Samuels MH. Psychiatric and cognitive manifestations of hypothyroidism. Curr Opin Endocrinol Diabetes Obes. 2014;21(5):377-383. doi:10.1097/MED.0000000000000089

7. Feldman AZ, Shrestha RT, Hennessey JV. Neuropsychiatric manifestations of thyroid disease. Endocrinol Metab Clin North Am. 2013;42 (3):453-476. doi:10.1016/j.ecl.2013.05.005

8. De Leo S, Lee SY, Braverman LE. Hyperthyroidism. Lancet. 2016;388(10047):906-918. doi:10.1016/S0140-6736(16)00278-6

9. Chaker L, Bianco AC, Jonklaas J, Peeters RP. Hypothyroidism. Lancet. 2017;390(10101):1550-1562. doi:10.1016/S0140-6736(17) 30703-1

10. Kundra P, Burman KD. The effect of medications on thyroid function tests. Med Clin North Am. 2012;96(2):283-295. doi:10.1016/j. mona.2012.02.001

11. Vedal TSJ, Steen NE, Birkeland KI, et al. Free thyroxine and thyroidstimulating hormone in severe mental disorders: a naturalistic study with focus on antipsychotic medication. $J$ Psychiatr Res. 2018;106:74-81. doi:10.1016/j.jpsychires.2018.09.014

12. Geddes JR, Miklowitz DJ. Treatment of bipolar disorder. Lancet. 2013;381(9878):1672-1682. doi:10.1016/S0140-6736(13)60857-0

13. Findling RL, Pathak S, Earley WR, Liu S, DelBello MP. Efficacy and safety of extended-release quetiapine fumarate in youth with bipolar depression: an 8 week, double-blind, placebo-controlled trial. J Child Adolesc Psychopharmacol. 2014;24(6):325-335. doi:10.1089/cap. 2013.0105
14. Peuskens J, Link CG. A comparison of quetiapine and chlorpromazine in the treatment of schizophrenia. Acta Psychiatr Scand. 1997;96 (4):265-273.

15. Poutanen O, Iso-Koivisto E, Tyolajarvi M, Leinonen E. Quetiapineassociated hypothyroidism in young female patients: a report of three cases. Pharmacopsychiatry. 2010;43(6):237-239. doi:10.1055/s0030-1261880

16. Kontaxakis VP, Karaiskos D, Havaki-Kontaxaki BJ, Ferentinos P, Papadimitriou GN. Can quetiapine-induced hypothyroidism be reversible without quetiapine discontinuation? Clin Neuropharmacol. 2009;32(5):295-296. doi:10.1097/WNF.0b013e3181a8cbcc

17. Feret BM, Caley CF. Possible hypothyroidism associated with quetiapine. Ann Pharmacother. 2000;34(4):483-486. doi:10.1345/ aph.19186

18. Liappas J, Paparrigopoulos T, Mourikis I, Soldatos C. Hypothyroidism induced by quetiapine: a case report. $J$ Clin Psychopharmacol. 2006;26(2):208-209. doi:10.1097/01.jcp.000020 3227.56494.ff

19. Parmentier T, Sienaert P. The use of triiodothyronine (T3) in the treatment of bipolar depression: a review of the literature. $J$ Affect Disord. 2018;229:410-414. doi:10.1016/j.jad.2017.12.071

20. Lopez-Munoz F, Alamo C. Active metabolites as antidepressant drugs: the role of norquetiapine in the mechanism of action of quetiapine in the treatment of mood disorders. Front Psychiatry. 2013;4:102. doi:10.3389/fpsyt.2013.00102

21. Bunevicius R, Steibliene V, Prange AJ Jr. Thyroid axis function after in-patient treatment of acute psychosis with antipsychotics: a naturalistic study. BMC Psychiatry. 2014;14:279. doi:10.1186/s12888-0140279-7

22. Kelly DL, Conley RR. Thyroid function in treatment-resistant schizophrenia patients treated with quetiapine, risperidone, or fluphenazine. J Clin Psychiatry. 2005;66(1):80-84.

23. de Leon J. Glucuronidation enzymes, genes and psychiatry. Int $J$ Neuropsychopharmacol. 2003;6(1):57-72. doi:10.1017/S146114570 3003249

24. Zepf FD, Vloet TD, Polier GG, et al. No association between affective and behavioral dysregulation and parameters of thyroid function in youths. $J$ Affect Disord. 2011;134(1-3):478-482. doi:10.1016/j. jad.2011.05.040
Neuropsychiatric Disease and Treatment

\section{Publish your work in this journal}

Neuropsychiatric Disease and Treatment is an international, peerreviewed journal of clinical therapeutics and pharmacology focusing on concise rapid reporting of clinical or pre-clinical studies on a range of neuropsychiatric and neurological disorders. This journal is indexed on PubMed Central, the 'PsycINFO' database and CAS, and

\section{Dovepress}

is the official journal of The International Neuropsychiatric Association (INA). The manuscript management system is completely online and includes a very quick and fair peer-review system, which is all easy to use. Visit http://www.dovepress.com/testimonials.php to read real quotes from published authors. 